\title{
Hypothesis Article: COVID-19 unexplained mortality in the young adults: could it be due to ACE2 polymorphisms?
}

\author{
Mina Kelleni ${ }^{1}$ \\ ${ }^{1}$ Minia University
}

June 17, 2020

Fang and his colleagues have suggested to investigate the genetic predisposition for an increased risk of SARS-CoV-2 infection and they've proposed an explanation that might be linked to its receptor ACE2 polymorphisms ${ }^{1}$. The author agrees with their suggestion and would like to discuss it in a more elaborative manner.

ACE2 polymorphisms and its induced mutations have been previously linked to enhanced susceptibility of heart diseases including coronary heart disease, myocardial infarction as have been revealed both clinically and experimentally ${ }^{2,3}$. Further, The ACE2 rs4646188 variant was suggested as a potential and optimal genetic susceptibility marker for essential hypertension, dyslipidemia and its related ischemic stroke ${ }^{4}$. Similarly, three ACE2 variants (rs4240157, rs4646155, and rs4830542) were found to be associated with essential hypertension and hypertension-related atrial fibrillation and left atrial remodeling ${ }^{5}$ Further, genetic variants in the ACE2 gene have been suggested to be associated with left ventricular mass, septal wall thickness and left ventricular hypertrophy in hemizygous men ${ }^{6}$. Noteworthy, genetic variants in the ACE2 gene were significantly associated with diastolic blood pressure responses to cold stress in the Chinese female population ${ }^{7}$. The author would like to suggest that It might be probable that ACE2 polymorphisms in the lungs could be one of the causes linked to a higher morbidity and/or mortality rate encountered in some groups of COVID-19 patients rather than the drugs suggested by Fang and his colleagues which have been refuted ${ }^{8}$. Further, these polymorphisms might also be one of the answers why some young, apparently healthy adults have been deceased while some very old patients have been rescued. The author recommends examining COVID-19 consented autopsies to explore this hypothesis as it might help us to develop some genetic tests to warn those more susceptible individuals exempt vulnerable health care professionals from duty.

Conflict of interests:

The author has no conflicts of interest to declare.

Funding:

None.

References:

1. Fang L, Karakiulakis G, Roth M. Are patients with hypertension and diabetes mellitus at increased risk for COVID-19 infection? The Lancet Respiratory Medicine 2020;8:e21.

2. Wang W, Patel VB, Parajuli N, et al. Heterozygote loss of ACE2 is sufficient to increase the susceptibility to heart disease. Journal of Molecular Medicine 2014;92:847-58.

3. Yang W, Huang W, Su S, et al. Association study of ACE2 (angiotensin I-converting enzyme 2) gene polymorphisms with coronary heart disease and myocardial infarction in a Chinese Han population. Clin 
Sci (Lond) 2006;111:333-40.

4. Pan Y, Wang T, Li Y, et al. Association of ACE2 polymorphisms with susceptibility to essential hypertension and dyslipidemia in Xinjiang, China. Lipids Health Dis 2018;17:241.

5. Luo Y, Liu C, Guan T, et al. Association of ACE2 genetic polymorphisms with hypertension-related target organ damages in south Xinjiang. Hypertens Res 2019;42:681-9.

6. Lieb W, Graf J, Gotz A, et al. Association of angiotensin-converting enzyme 2 (ACE2) gene polymorphisms with parameters of left ventricular hypertrophy in men. Results of the MONICA Augsburg echocardiographic substudy. J Mol Med (Berl) 2006;84:88-96.

7. Huang J, Chen S, Lu X, et al. Polymorphisms of ACE2 are associated with blood pressure response to cold pressor test: the GenSalt study. Am J Hypertens 2012;25:937-42.

8. ACEIs, ARBs, Ibuprofen linked to COVID-19: The other side of the broken mirror. 2020, June 2,. at https://www.authorea.com/users/318758/articles/456017-aceis-arbs-ibuprofen-linked-to-covid-19-theother-side-of-the-broken-mirror?commit=c85d790e9c1c2a3e698675ed1798efe534abdfa7.) 